\title{
CENTRAL LONDON OPHTHALMIC HOSPITAL
}

\author{
Report of Squint Department,
}

September, 1932-1933

BY

Sigrid PeARSon, M.R.C.S., L.R.C.P., D.O.M.S.

AND

\author{
Sheila mayou \\ (SENIOR ORTHOPTIC ASSISTANT)
}

DURING the first year's work in the department 171 cases have been seen. The number of patients treated increased gradually as more instruments became available, and at the end of the year 39 patients per week were under treatment. To show the increase of work in the department, in September, 1932, there were 96 attendances, and in September, 1933, 280 attendances. These numbers include patients on homework and occlusion, who attend occasionally for supervision, and those under treatment.

The department is open from 2-5 p.m. every day except Saturday, and each patient receives 30 minutes treatment.

Methods employed.-The instruments used in the department have been the synoptophore, the myoscope, and the strabismuscope; homework has been done chiefly on the cheiroscope, though in certain cases the stereoscope and amblyoscope have been used. Our method with amblyopias has almost invariably been to start treatment with constant and total occlusion, lessening the size and opacity of the occluder as the vision improves. We have not found intermittent occlusion satisfactory and it has not been possible so far to experiment with intensive stimulation of amblyopic maculae. It has always been our object to obtain fusion with some power of duction before any case goes to the operating theatre, but on account of the length of our waiting-list and the inability of patients to attend for a prolonged period, we have not always been able to manage this. We have made a point of rather detailed and accurate history taking, as we feel that in this way some useful light may be thrown on the aetiology of squint and the type of child in which a good result may be obtained.

Analysis of cases.-We realize that our present numbers do not permit of wide generalizations of any kind, nevertheless, one or two points of interest appear. Of the total number of cases seen, 34 were refused as unsuitable for treatment. With the exception of a small proportion of failures, generally due to inability to attend or lack of home co-operation, an analysis of our cases is as follows :- 


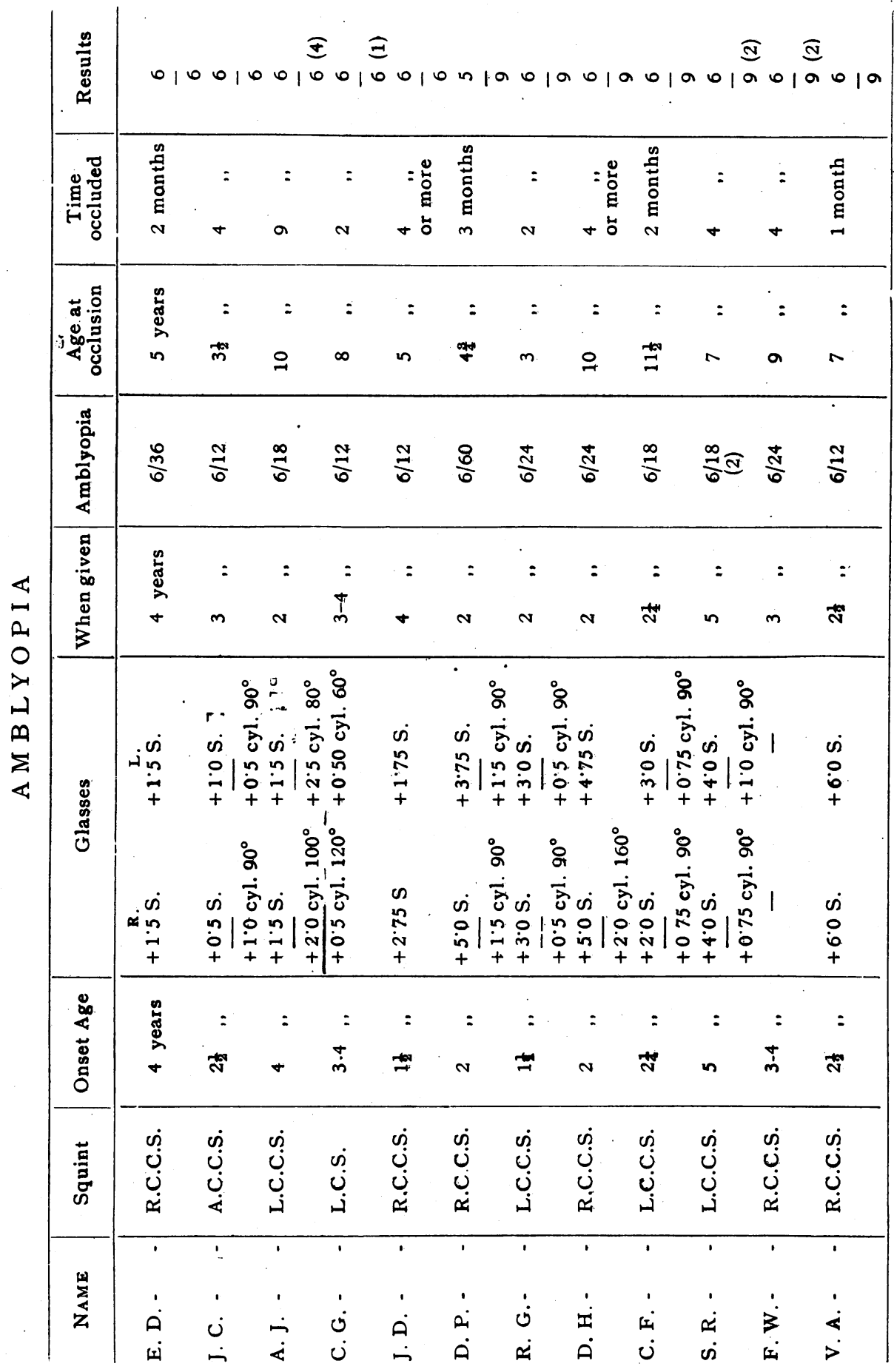


Central London Ophthalmic Hospital

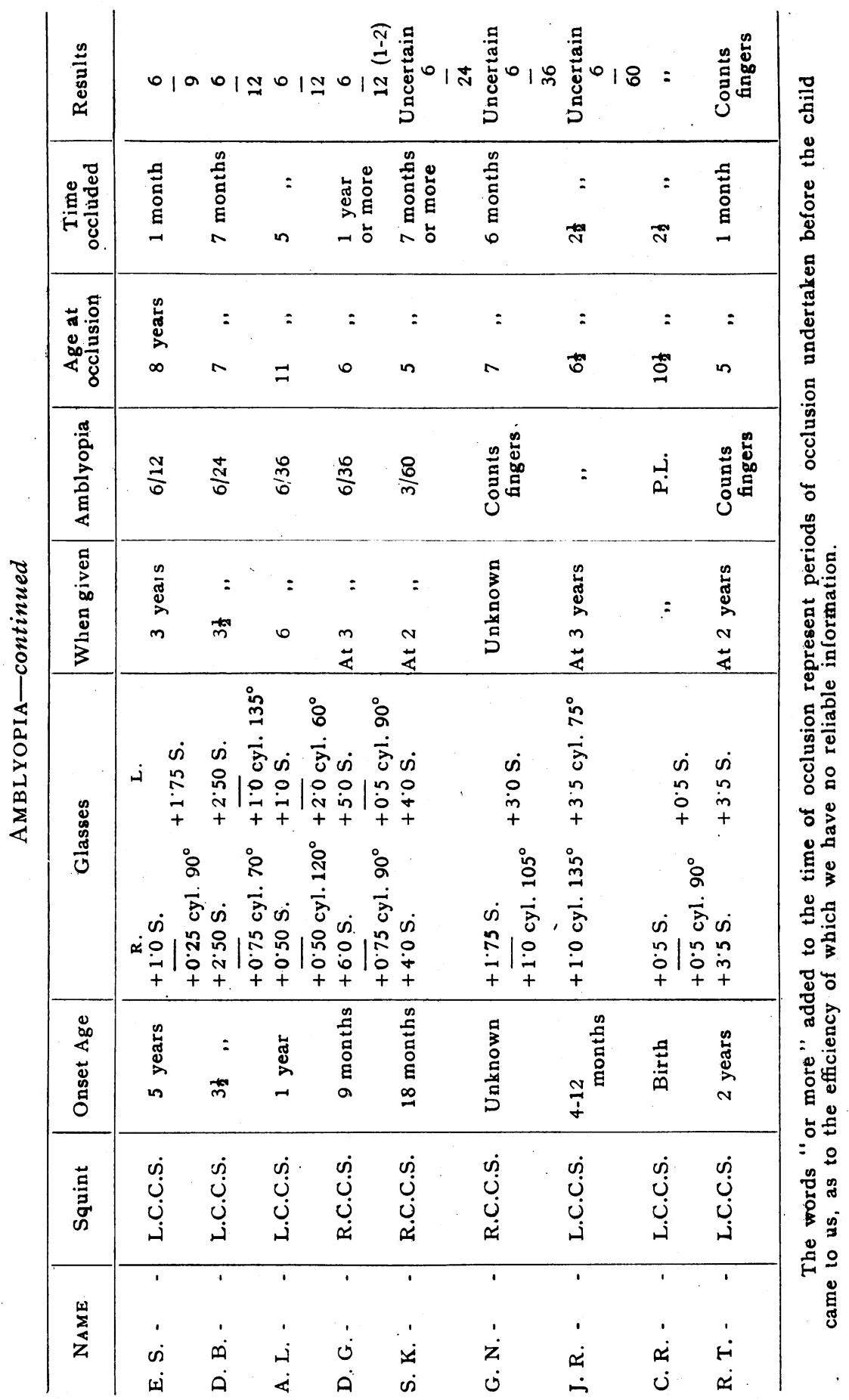


Amblyopias.-In the detailed analysis of those amblyopias which we have treated, it will be seen how extremely difficult it is to overcome amblyopia resultant from a squint occurring in the first two years of life, if occlusion is delayed until after the child is 5 years of age, whereas occlusion undertaken under 5 years of age has rapidly resulted in the recovery of good vision. Two or three cases of rapid and successful recovery in older children with a high degree of astigmatism seem to suggest that these squints should probably be placed in a separate category.

Discharged cases.-The word "cure" used below in reference to discharged cases denotes the possession of the three grades of binocular vision, i.e., simultaneous perception, fusion and stereopsis; the angle of deviation on discharge in these cases was $0^{\circ}$ on the synoptophore and there was no muscular imbalance shown with either the Maddox Rod or Wing Test.

Seven cases of squint we consider as cured without operation. These had never at any time when under observation more than $10^{\circ}$ of squint in glasses, and, with the exception of one, required on an average 15 lessons of 30 minutes each.

Eighteen of our cases have undergone operation in addition to orthoptic treatment, and of these, nine can be considered as cured, whilst the remaining nine are cosmetically straight and lacking only in stereopsis. It is worth while noting that of these only cosmetically satisfactory cases, none-with the exception of a case of congenital nystagmus-had had pre-operative treatment. We feel, however, that much more investigation is necessary before we can dogmatize about the relative importance to success of the three factors : amount of treatment, type of operative procedure, and type of squint.

All the patients discharged as cured are required to come up for frequent observation, as we feel that information on the subject of possible relapses should be obtained.

Asthenopia.-Four cases of asthenopia have been treated with excellent results; in each case the convergence has been increased from $10^{\circ}$ to $35^{\circ}$.

Cases under treatment.-At the present moment we have 39 cases under treatment, of which a certain number are still attending after eight months. We feel that the last fact is an important one as it serves to show what an immense amount of work in the present state of our knowledge may be necessary to effect a "cure."

Cases not accounted for in this analysis are still on the waitinglist, either on account of amblyopia in process of cure, too tender years, or lack of accommodation in the department. 